\title{
A Quantitative Study of the Microstructure and Biochemistry of the Medial Meniscal Horn Attachments
}

\author{
Diego F. Villegas, ${ }^{1}$ Tara A. Hansen, ${ }^{1}$ Dong F. Liu, ${ }^{2}$ and Tammy L. Haut Donahue ${ }^{1}$ \\ ${ }^{1}$ Department of Mechanical Engineering, Michigan Technological University, 1400 Townsend Dr., Houghton, MI 49931, USA; \\ and ${ }^{2}$ Portage Health System, Houghton, MI, USA
}

(Received 16 April 2007; accepted 31 October 2007; published online 13 November 2007)

\begin{abstract}
Little quantitative data is available on the structure of meniscal attachments. Therefore, as an aid to designing meniscal replacements as well as a possible explanation for mechanical behavior, this study was designed to further the knowledge of the microstructure and biochemistry of native meniscal attachments. Bovine medial meniscal attachments (the external ligamentous portion as well as the transition zones at the bony insertion) were removed and prepared for microstructural evaluation. After embedding in paraffin, the samples were sliced on a microtome and stained for quantitative analysis. The anterior and posterior insertion sites are known to contain three zones: subchondral bone, calcified fibrocartilage, and uncalcified fibrocartilage. Additionally, others have shown that the anterior insertion site contains a ligamentous zone. The insertion zones were further divided into proximal, middle, and distal zones. The posterior attachment's insertion site had a significantly greater thickness of interdigitations, subchondral bone, uncalcified fibrocartilage, and calcified fibrocartilage zone thickness compared to the anterior attachment insertion. The anterior attachment's insertion had the greatest GAG fraction in each zone when compared to the posterior attachment's insertion. GAG fraction decreased from the meniscus to the subchondral bone. Both GAG fraction and normalized thickness varied within a given zone, decreasing from the distal to proximal regions in both the anterior and posterior attachments' insertion zones. Crimp frequency of the collagen fibrils in the external ligamentous portion of the tissue was homogeneous along the length. The findings from this study agree with previously published material property data on the medial meniscal attachments, and could be used in the future to design methods of attachment for tissue engineered replacement menisci.
\end{abstract}

Keywords-Collagen orientation, Glycosaminoglycan, Meniscus, Enthesis.

Address correspondence to Tammy L. Haut Donahue, Department of Mechanical Engineering, Michigan Technological University, 1400 Townsend Dr., Houghton, MI 49931, USA. Electronic mail: thdonahu@mtu.edu

\section{INTRODUCTION}

Menisci are fibrocartilagenous structures in the knee joint that aid in joint lubrication and stability. ${ }^{4,40}$ Menisci are frequently torn due to both degeneration and traumatic injuries. Many of these tears do not heal due to their complexity or location in the avascular regions of the meniscus. When the tears do not heal, a complete or partial meniscectomy is the common treatment. Short-term clinical studies indicate improvements in patient pain. ${ }^{15}$ However, long term studies have shown that a meniscectomy can lead to degenerative arthritis of the knee. ${ }^{15}$ Tissue engineered (TE) menisci are currently being developed as a replacement for damaged menisci following a meniscectomy.

Helping to prevent premature arthritis in meniscectomy patients, TE menisci could be a potential solution when tears do not heal. ${ }^{42}$ The success of TE menisci in the human body depends on their ability to restore normal meniscal function. It is believed that material properties, geometry, and attachment to the tibial plateau affect meniscal function. ${ }^{39}$ Previous experimental and theoretical studies have shown that meniscal attachment strength affects meniscal stability and function. ${ }^{5,12,22,24}$

Anchoring the menisci at the anterior and posterior horns, the meniscal attachments are primarily composed of Type I collagen fibrils extending from the main body of the meniscus into the tibial plateau. ${ }^{33}$ The attachments are often referred to as insertional ligaments. They are defined as the part of the meniscus extending from the edge of the meniscal horn to the bony insertion on the tibia. ${ }^{18,22}$ Meniscal attachments are made up of water, proteoglycans, and collagen, which is oriented parallel to the direction of loading. ${ }^{47}$ Serving as the transition from meniscal fibrocartilage into subchondral bone, they act as the primary anchor for the menisci to the tibial plateau. The meniscal attachment consists of an external, visible ligamentous 
structure, which then inserts into the underlying bone. In a study done in rabbits, Gao et al. suggested that the anterior attachment is primarily in tension, while the posterior attachment is subjected to both tension and compression. ${ }^{18}$ The authors have previously quantified the time-dependent and failure properties of the anterior and posterior medial meniscal attachments. ${ }^{31,46}$ While no significant differences in the creep or stress-relaxation properties between the anterior and posterior medial meniscal attachment were found, the ultimate strain in tension and tensile modulus were significantly different. The anterior attachment had a significantly lower tensile modulus and ultimate strain.

The zones of the medial meniscal attachment's insertion into the subchondral bone have been previously identified. ${ }^{17-20,22}$ In both rabbit and man, the anterior and posterior insertion sites have been identified to contain three zones: uncalcified fibrocartilage, calcified fibrocartilage, and subchondral bone. The anterior insertion site contains an additional zone referred to as a ligamentous zone. ${ }^{8,18}$ Limited research has been conducted to quantitatively describe the microstructure in these transition zones. Benjamin et al., showed quantitatively that the thickness of both the uncalcified and calcified fibrocartilage in human medial meniscal attachments were greater in the posterior attachments compared to the anterior attachment. ${ }^{8}$ With regard to the composition of meniscal attachments, Gao et al., 2000 determined the distribution of types I, II, and X collagen in tibial insertion sites of medial meniscus by immunohistochemistry. ${ }^{16}$ Aggrecan is a proteoglycan found in fibrocartilage that support compressive loads ${ }^{9}$ due to the high osmotic pressure created by its GAG chains. ${ }^{25}$ Previous researchers have suggested that meniscal attachments' insertion sites are subjected to compressive loads, ${ }^{16}$ however, no measurement of GAG fraction has been made to date. The distribution of GAGs across the insertion site will provide a better understanding of how meniscal attachments carry and distribute the loads into the tibia. To further understand how the microstructure and biochemistry may relate to function, we seek (1) to determine the glycosaminoglycan (GAG) fraction of the anterior and posterior medial meniscal attachments' insertion zones, (2) to determine the collagen fibril crimp frequency in the external attachment, and (3) to provide normalized thickness data on each zone of the attachments' insertion into the bone. A better understanding of the microstructure and composition could provide further insight into these transitional tissues, allowing for better TE replacement attachments to be designed. In addition, these data may help explain the mechanical response of attachments when the meniscus is loaded.

\section{MATERIALS AND METHODS}

\author{
Specimen Preparation
}

The microstructure of the anterior and posterior medial meniscal horn attachments were studied in both knees of nine bovines that were 2-years old (skeletally mature). During dissection the knees were visually inspected. If any pre-existing damage or signs of osteoarthritis was found, the knees were rejected from the study. The knee joints with attached femur and tibia were obtained from a local abattoir and frozen within $24 \mathrm{~h}$ after death. The knee joints were kept frozen until dissection.

After dissection of the knee joint and removal of the femur, the medial meniscal horn attachments were isolated. The attachment consists of the external ligament portion, and the transitional insertion into the bone (Fig. 1). The width, depth, and proximal (P) and distal (D) surface length of the external ligament portion were measured using calipers. It should be noted that the external ligament portion has previously been shown to have a longer proximal surface length than the distal surface. ${ }^{31}$ The external ligament portion of the attachment was defined as extending from the edge of the meniscal body to its insertion into the bone (Fig. 1). The medial meniscus was bisected and the attached horn (either anterior or posterior) was removed with the underlying bone using a band saw. The attachments are in such close proximity that removal of the medial anterior attachment destroys the lateral anterior attachment thus the lateral anterior attachment was not included in the study. The bovine lateral posterior attachment inserts into the femur and thus was not studied. The meniscal attachment block was sliced into $5 \mathrm{~mm}$ sections, parallel to the collagen fibrils and prepared for histological evaluation (Fig. 1).

The samples were fixed in $10 \%$ neutral buffered formalin for $48 \mathrm{~h}$ and then decalcified in $10 \%$ formic acid for 8 days. After removal from formic acid, the samples were fixed again in $10 \%$ formalin and dehydrated in increasing concentrations of ethanol. Following clearing of the samples in xylene, the samples were infiltrated and embedded in paraffin. The paraffin-embedded blocks were sliced on a microtome (Shandon AS325, Thermo Electron Corp., Waltham, MA) to obtain $10 \mu \mathrm{m}$ slices at $200 \mu \mathrm{m}$ intervals parallel to the collagen fibrils. Half of the slides were stained with safranin $\mathrm{O}$ for GAG analysis and collagen crimp frequency, and the other half were stained with hematoxylin and eosin $(\mathrm{H} \& \mathrm{E})$ and alcian blueperiodic acid schiff (AB-PAS) for microstructural evaluation. 


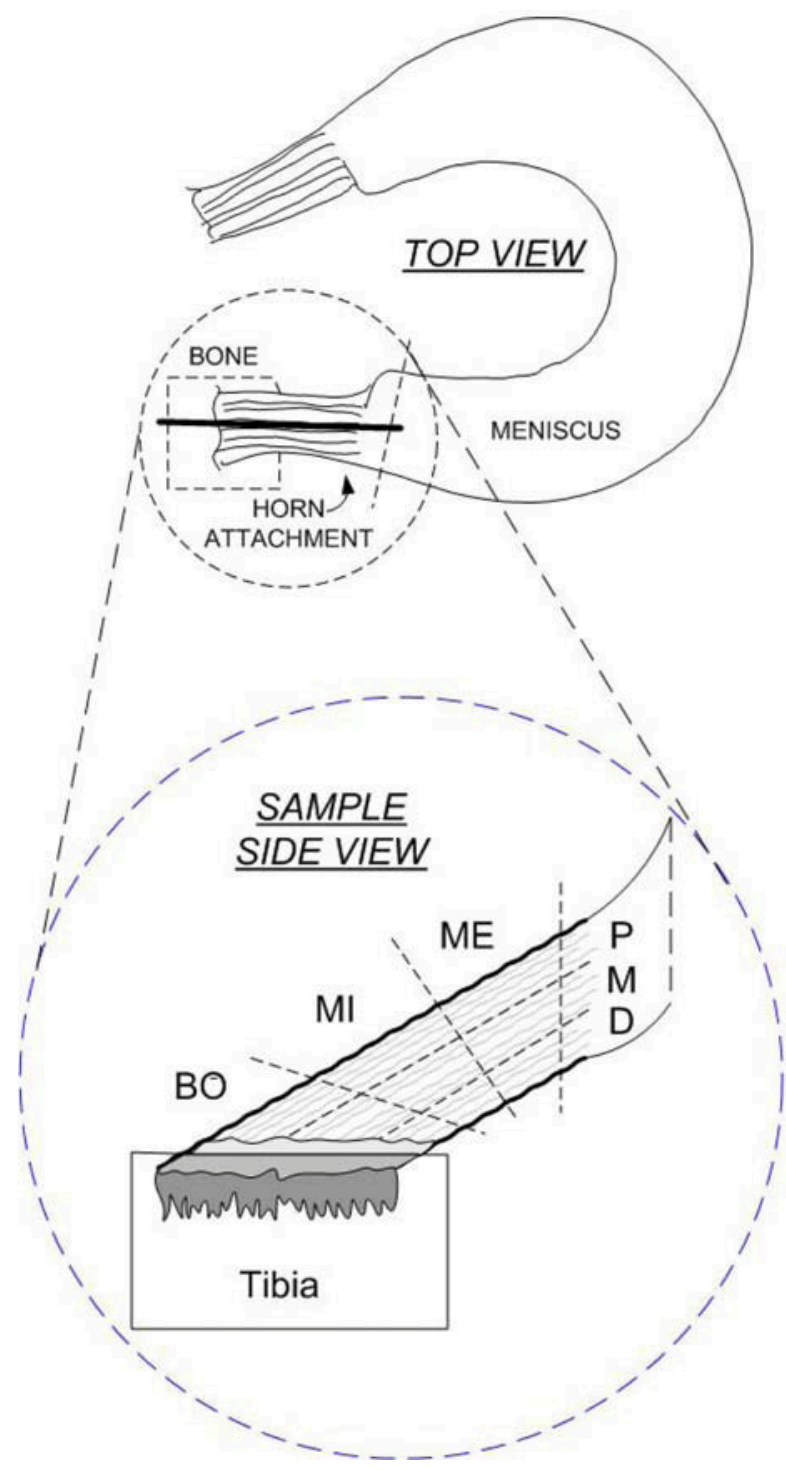

FIGURE 1. Anatomical layout of meniscus showing external ligament portion of the horn attachment, and the location of the bone block taken to make histological slices. Side view shows how external ligament was divided into meniscus (ME), midsubstance (MI), and bony insertion (BO) zones as well as showing the proximal $(P)$, middle $(M)$, and distal $(D)$ regions within each zones. The transition zones into the tibia are also shown with interdigitations.

\section{Zones of the Medial Menisci Attachments' Insertions}

The H\&E and AB-PAS stained slides were analyzed using a light microscope (Olympus CX4, Melville, NY), a digital camera (Canon PowerShot G6, Lake Success, NY), and an image analysis program created in Matlab v7.1 (Mathworks, Inc., Natick, MA). The program determined the GAG fraction on the pictures using the pixel channel (red (R), green $(\mathrm{G})$, and blue (B)). The program allowed for opening many pictures quickly, saving and tabulating the data automatically. The thicknesses of the ligamentous zone (anterior attachment only), uncalcified fibrocartilage, calcified fibrocartilage, and subchondral bone and the frequency and thickness of the interdigitations were outlined and measured as previously described ${ }^{8,18}$ (Fig. 2). The total thickness of the anterior attachment insertion was the sum of all four zones for the anterior insertion, and the posterior attachment insertion thickness was the sum of the three defined zones. The insertional ligamentous zone in the anterior attachment extends from the wavy collagen fibrils of the external ligament to the first series of chondrocytes in the uncalcified fibrocartilage. The frequency of interdigitations was calculated by dividing the number of interdigitations over the length that they spanned. The subchondral bone thickness was measured by calculating the mean distance from the interdigitations to the beginning of the trabecular bone. While these zones are well-defined by others, to ensure repeatability between attachments in the distinction between these zones, three blind users quantified the data (KJ, DV, TH). The data collected by all three users came to the same statistical conclusion, hence, the data from only one user is presented (DV). Each user analyzed coded samples. Since the sections are decalcified for thin slicing, it was not possible to stain for calcium to help distinguish transitions from calcified to uncalcified zones. Von Kossa calcium staining was completed on thicker sections that had not been decalcified, however the thicker sections prevented viewing of the interdigitations.

The thickness of the uncalcified fibrocartilage and calcified fibrocartilage were measured as described in Gao et al., 1996. ${ }^{17}$ Briefly, the thickness of the uncalcified fibrocartilage was defined as the distance between the tidemark and the most distally located series of chondrocytes. The tidemark was characterized as the border between uncalcified and calcified fibrocartilage. The cement line was defined as the uneven border between the calcified fibrocartilage and subchondral bone. This uneven border results in interdigitations between these zones, thereby increasing the contact area between the calcified fibrocartilage and subchondral bone. The mean distance between the tidemark and interdigitations was described as the thickness of the calcified fibrocartilage. The thickness of interdigitations was defined as the mean distance between the peak and trough of the interdigitations. Proximal, middle and distal thicknesses of each zone were normalized with respect to the corresponding external ligament length. The middle length was taken as the average between proximal and distal length.

\section{GAG Quantification}

The safranin $O$ stained slides were analyzed for glycosaminoglycan (GAG) fraction using a light 


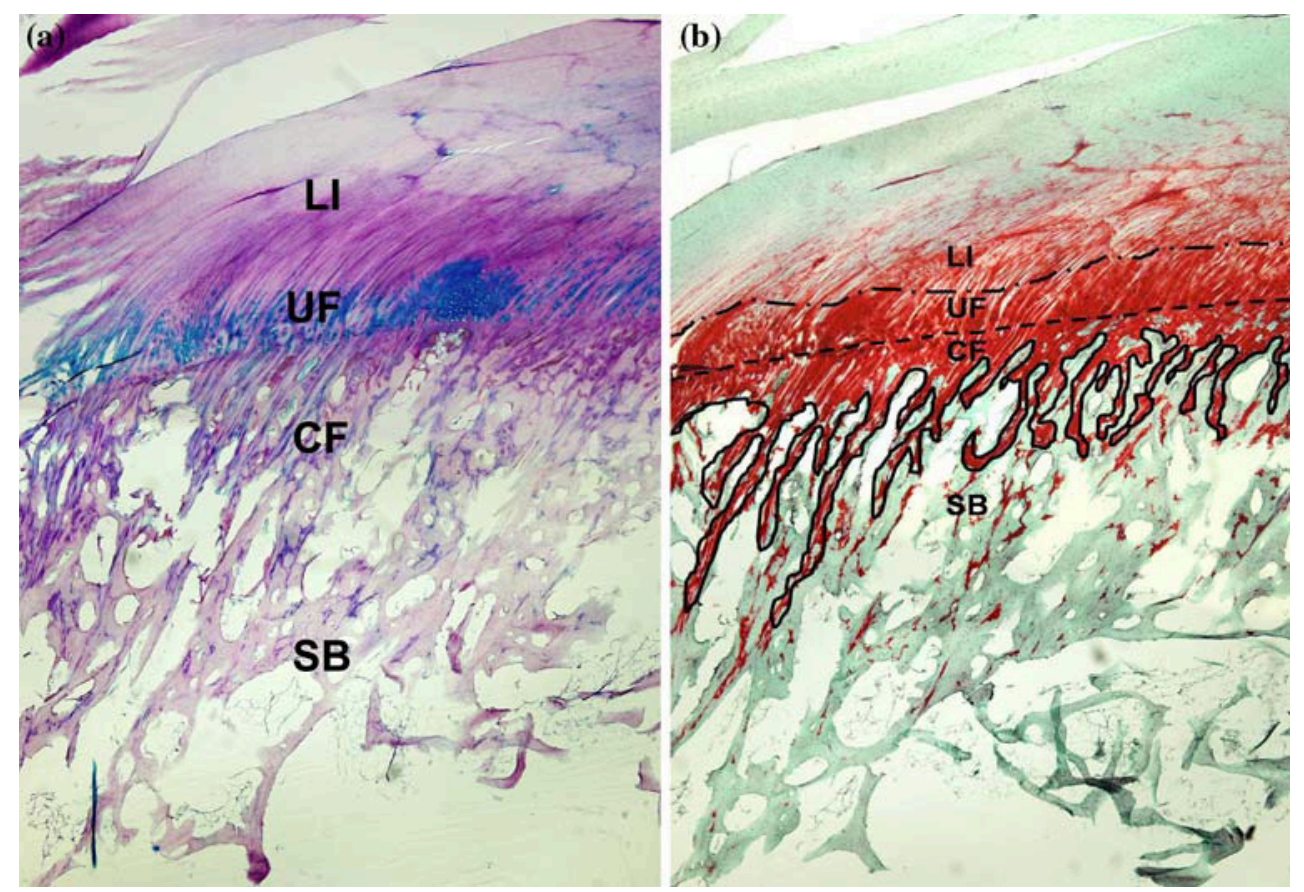

FIGURE 2. (a) Anterior medial meniscal attachment transition zones stained with H\&E/AB-PAS and used for microstructural measurements (b) Anterior medial meniscal attachment transition zones stained with Safranin $O$ for GAG fraction measurements. Interdigitations are overlaid on the slide. Key: SB-Subchondral Bone; CF-Calcified Fibrocartilage; UF-Uncalcified Fibrocartilage; LI-Ligamentous zone.

microscope (Olympus CX4, Melville, NY), a digital camera (Canon PowerShot G6, Lake Success, NY), and an image analysis program created in Matlab (Mathworks, Inc., Natick, MA). The binding intensity of the safranin $O$ stain was correlated to the GAG fraction similarly to Huang et al., 2002. ${ }^{26}$ In short, the intensities of the red (R), green $(\mathrm{G})$, and blue (B) channels were assigned values from 0 to 255 . The proportion of red color to all of the colors primarily represented the safranin $\mathrm{O}$ binding and was calculated using the equation $r=\mathrm{R} /\left(\mathrm{R}^{2}+\mathrm{G}^{2}+\mathrm{B}^{2}\right)^{1 / 2}$. The higher the $r$ value represented the greater GAG fraction. Each attachment's insertion was divided into its zones, four anterior attachment zones and three posterior attachments zones. Each respective attachment zone was then divided into three regions: distal, middle, and proximal (Fig. 1). The GAG fraction in each region was measured.

\section{Collagen Fibril Crimp Frequency}

The external ligamentous portion of the attachments was divided into the three longitudinal zones (ME, MI, and $\mathrm{BO}$ ) and three regions $(\mathrm{P}, \mathrm{M}$, and $\mathrm{D})$ to divide each sample into nine sections (Fig. 1). ME and $\mathrm{BO}$ zones were taken where the fraction of GAG diminishes (no red stain). To measure collagen crimp frequency a Zeiss Apotome Microscope equipped with a Differential Interference Contrast (DIC) lens was used. Pictures were taken in each section with a digital camera and analyzed with a custom-made program in Matlab v7.1 (Mathworks, Inc., Natick, MA). The users chose three successive crimps at four different locations on the picture to obtain 12 measurements of crimp period, and calculated the frequency of the crimps. The program measured the crimp period (see Fig. 3). The data is presented in crimps $/ \mathrm{mm} \pm \mathrm{SD}$.

\section{Statistics}

Paired $t$-tests were used to compare differences in external ligament dimensions, thickness of attachment insertion zones, frequency of interdigitations, and GAG fraction between the anterior and posterior attachment insertions. ANOVA was used with Bonferroni post-hoc tests for assessing differences between proximal, middle, and distal regions for the thickness of the attachment zones and GAG fractions in the anterior and posterior attachments, and collagen crimp frequency in the external attachment. Linear regression was used to determine trends from the proximal to distal regions or from the ligamentous zone to subchondral bone. 


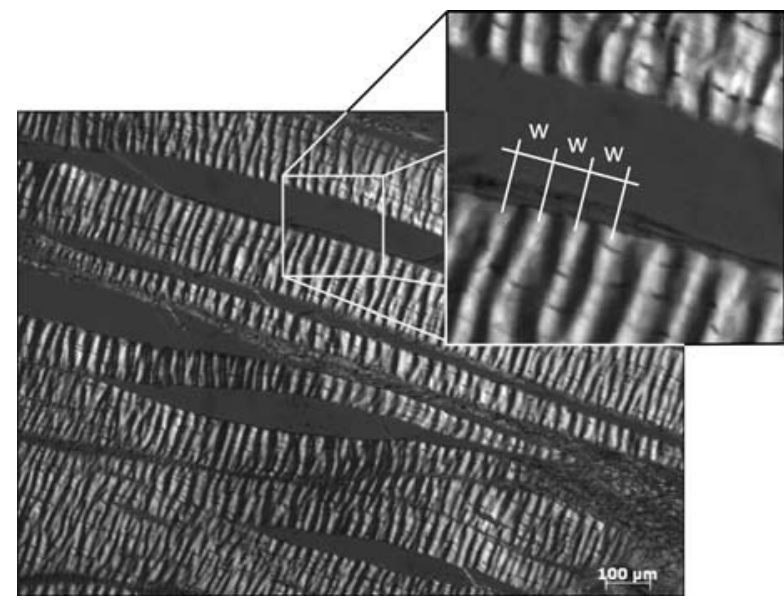

FIGURE 3. Representative image taken with a Differential Contrast Microscopy of collagen crimp frequency in meniscal attachment. The period (w) was taken as the distance between two consecutive peaks.

\section{RESULTS}

\section{External Ligament Attachment}

The posterior ligament was significantly wider and shorter in length than the anterior ligament $(p<0.05)$. The anterior external ligament had a width of $13.9 \pm 1.2$, a proximal length of $25.7 \pm 1.6$, a distal length of $13.3 \pm 1.2$, and a depth of $4.4 \pm 0.3 \mathrm{~mm}$. The posterior external ligament had a width of $17.9 \pm 1.7$, a proximal length of $18.8 \pm 1.4$, a distal length of $12.1 \pm 0.5$, and a depth of $4.34 \pm 0.33 \mathrm{~mm}$. All values are represented as mean $\pm \mathrm{SD}$.

\section{Microstructure of the Meniscal Attachments' Insertions into Bone}

The posterior attachment's insertion site had a significantly greater thickness of interdigitations, subchondral bone, uncalcified fibrocartilage, and calcified fibrocartilage zone thickness compared to the anterior attachment insertion (Table 1, Fig. 2b). Additionally, all zones were significantly different with respect to each other, except for UF and CF in both attachments $(p>0.05)$ (Table 1). There was a gradual but significant decrease in the thickness of the zones from the ligamentous zone to the calcified fibrocartilage zone (Table 1).

Breaking each zone into three transverse regions $(\mathrm{P}$, $\mathrm{M}$, and D) and measuring the zone thicknesses in each region, revealed significant differences between all regions $(p<0.05$, Table 1). The attachment's insertion zone normalized thicknesses and interdigitation thicknesses significantly increased from the proximal to the distal regions (Table 1). On the other hand, the frequency of interdigitations per millimeter for both medial meniscal attachments look similar between proximal, middle, and distal regions with values of $3.9 \pm 0.6,4.0 \pm 0.7$, and $4.0 \pm 0.6$ interdigitations/ $\mathrm{mm}$, respectively for the anterior, and $4.0 \pm 1.1$, $4.4 \pm 1.2$, and $4.5 \pm 1.2$ interdigitations $/ \mathrm{mm}$ for the posterior attachment. The frequency of interdigitations was significantly greater in the posterior attachment $(p<0.05)$.

Collagen fibril crimp frequency showed no significant differences between attachments and longitudinal zones (ME, MI, BO) in both attachments. With the

TABLE 1. Normalized thickness of the anterior and posterior medial meniscal attachment zones.

\begin{tabular}{|c|c|c|c|c|c|c|c|c|}
\hline & \multicolumn{4}{|c|}{ Anterior attachment $\mathrm{e}^{\mathrm{e}}$} & \multicolumn{4}{|c|}{ Posterior attachment } \\
\hline & Proximal & Middle $^{f}$ & Distal $^{\mathrm{f}, \mathrm{g}}$ & Average & Proximal & Middle $^{f}$ & Distal $^{\mathrm{f}, \mathrm{g}}$ & Average \\
\hline Total & $11.8 \pm 2.3$ & $16.0 \pm 3.3$ & $24.17 \pm 5.3$ & $17.3 \pm 6.4$ & $14.7 \pm 4.1$ & $19.8 \pm 3.5$ & $26.0 \pm 4.7$ & $20.1 \pm 6.2$ \\
\hline Ligamentous (L) & $1.9 \pm 0.4^{\mathrm{a}}$ & $2.8 \pm 0.7^{a}$ & $4.7 \pm 1.1^{\mathrm{a}}$ & $3.1 \pm 1.4^{\mathrm{a}}$ & - & - & - & - \\
\hline $\begin{array}{l}\text { Uncalcified } \\
\text { fibrocartilage (UF) }\end{array}$ & $1.5 \pm 0.4$ & $2.2 \pm 0.5$ & $3.5 \pm 1.0$ & $2.4 \pm 1.1$ & $2.5 \pm 0.9$ & $3.3 \pm 1.0$ & $4.3 \pm 1.3$ & $3.4 \pm 1.3$ \\
\hline $\begin{array}{l}\text { Calcified } \\
\text { fibrocartilage (CF) }\end{array}$ & $1.3 \pm 0.4^{b}$ & $1.8 \pm 0.5^{b}$ & $2.7 \pm 1.0^{b}$ & $1.9 \pm 0.9^{b}$ & $2.3 \pm 0.8$ & $3.1 \pm 0.7$ & $4.1 \pm 1.1$ & $3.1 \pm 1.1$ \\
\hline $\begin{array}{l}\text { Subchondral } \\
\text { Bone (BO) }\end{array}$ & $5.9 \pm 2.0^{a, b, c}$ & $8.2 \pm 2.8^{\mathrm{a}, \mathrm{b}, \mathrm{c}}$ & $12.3 \pm 3.6^{a, b, c}$ & $8.8 \pm 3.9^{a, b, c}$ & $8.6 \pm 2.9^{\mathrm{a}, \mathrm{c}}$ & $11.9 \pm 3.2^{\mathrm{a}, \mathrm{c}}$ & $15.4 \pm 4.0^{\mathrm{a}, \mathrm{c}}$ & $12.0 \pm 4.4^{\mathrm{a}, \mathrm{c}}$ \\
\hline $\begin{array}{l}\text { Interdigitations } \\
\text { (INT) }\end{array}$ & $0.6 \pm 0.1^{\mathrm{a}, \mathrm{b}, \mathrm{c}, \mathrm{d}}$ & $0.9 \pm 0.2^{a, b, c, d}$ & $1.3 \pm 0.3^{\mathrm{a}, \mathrm{b}, \mathrm{c}, \mathrm{d}}$ & d $0.9 \pm 0.3^{a, b, c, d}$ & d $1.0 \pm 0.3^{\mathrm{a}, \mathrm{c}, \mathrm{d}}$ & $1.3 \pm 0.4^{\mathrm{a}, \mathrm{c}, \mathrm{d}}$ & $1.9 \pm 0.9^{\mathrm{a}, \mathrm{c}, \mathrm{d}}$ & d $1.4 \pm 0.7^{a, c, c}$ \\
\hline
\end{tabular}

Values are represented as mean $\pm \mathrm{SD}$.

${ }^{\text {a }}$ Statistically different than UF.

bStatistically different than L.

'Statistically different than CF.

${ }^{\mathrm{d} S}$ Statistically different than BO.

eStatistically different than posterior.

'Statistically different than proximal.

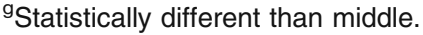


posterior medial attachment, no significant differences were found within regions, however, in the medial anterior attachment, the transverse regions (Proximal, Middle, Distal) had significant differences in the meniscus transition zone, bony insertion zone, and overall average (Table 2, Fig. 3).

\section{GAG Fraction in the Meniscal Insertions into Bone}

The anterior attachment's insertion zones had significantly higher GAG fraction than the posterior attachment's insertion zones. The GAG fraction significantly decreased from the anterior ligamentous attachment zone to the subchondral bone and from the posterior uncalcified fibrocartilage attachment zone to the subchondral bone $(p<0.05)$ (Table 3, Fig. 2b).

Significant differences in GAG fraction were seen between regions of the posterior attachments insertion. These data show that the proximal regions of the ligamentous, uncalcified fibrocartilage, and calcified fibrocartilage in the anterior and posterior meniscal attachments' insertions were lower in GAG fraction than the distal regions (Table 3). In contrast, the subchondral zone had no change in GAG fraction within a zone.

\section{DISCUSSION}

The medial meniscus is torn four times as often as the lateral meniscus. ${ }^{11,41}$ For this reason, the medial meniscal attachments were the focus of this study. This is the first data to quantify the thickness of all zones in medial meniscal attachments, and study variations within a zone from the proximal to distal regions within each attachment. Furthermore, we present for the first time the frequency of interdigitations, regional GAG fractions and collagen crimp frequency for both the anterior and posterior attachment. Based on studies that relate the collagen crimp frequency with time-dependent properties, ${ }^{6,7}$ we noted that our collagen crimp frequency results agree with time-dependent mechanical property testing which showed no difference between medial anterior and posterior attachments. ${ }^{31}$ The mechanical property tests did study an intact, entire attachment (external ligament portion and insertion zones) while the differences noted in collagen crimp frequency in the current study are based only on the external ligamentous portion. It does appear that both anterior and posterior attachments have a gradual decrease in GAG fraction from the external ligament portion to the subchondral bone. This study also showed that the normalized thickness is greater in the distal region in each attachment zone than the proximal region for both the anterior and the posterior attachment. In contrast, the proximal length of the external ligament portion is longer than the distal side. ${ }^{31}$ The clinical significance of this is currently unknown and requires further study.

In agreement with previous studies on the cruciate ligaments, ${ }^{19}$ this study found that the calcified

TABLE 2. Crimping of the anterior and posterior medial meniscal attachment zones.

\begin{tabular}{|c|c|c|c|c|c|c|c|c|}
\hline & \multicolumn{4}{|c|}{ Anterior attachment } & \multicolumn{4}{|c|}{ Posterior attachment } \\
\hline & Proximal $(\mathrm{P})$ & Middle (M) & Distal (D) & Average & Proximal $(\mathrm{P})$ & Middle (M) & Distal (D) & Average \\
\hline ME & $18.48 \pm 8.05$ & $27.72 \pm 4.52^{*}$ & $33.99 \pm 8.05^{*}$ & $27.29 \pm 8.54$ & $23.94 \pm 6.22$ & $24.17 \pm 7.16$ & $23.87 \pm 9.07$ & $24.00 \pm 7.18$ \\
\hline MI & $18.23 \pm 4.96$ & $26.88 \pm 5.49$ & $22.98 \pm 1.81$ & $22.70 \pm 5.43$ & $26.37 \pm 7.53$ & $27.11 \pm 5.25$ & $26.85 \pm 10.51$ & $26.78 \pm 7.93$ \\
\hline $\mathrm{BO}$ & $22.31 \pm 7.00$ & $30.94 \pm 8.36^{\star}$ & $34.75 \pm 11.17^{\star}$ & $29.73 \pm 10.50$ & $30.48 \pm 8.31$ & $32.51 \pm 9.79$ & $29.27 \pm 11.93$ & $30.76 \pm 10.19$ \\
\hline Average & $20.03 \pm 5.90$ & $29.21 \pm 6.83^{*}$ & $32.96 \pm 9.58^{\star}$ & & $27.44 \pm 7.91$ & $29.00 \pm 8.33$ & $27.50 \pm 10.98$ & \\
\hline
\end{tabular}

Values are represented as mean \pm SD (crimps per $\mathrm{mm}$ ). ME: Meniscus, MI: midsubstance, BO: Bony insertion.

* Significantly different than proximal region $(p<0.05)$.

TABLE 3. GAG fraction in the anterior and posterior medial meniscal attachment zones.

\begin{tabular}{|c|c|c|c|c|c|c|c|c|}
\hline & \multicolumn{4}{|c|}{ Anterior attachment } & \multicolumn{4}{|c|}{ Posterior attachment } \\
\hline & Proximal & Middle & Distal & Average & Proximal & Middle & Distal & Average \\
\hline Ligamentous & $0.73 \pm 0.10$ & $0.76 \pm 0.09$ & $0.80 \pm 0.08$ & $0.76 \pm 0.09$ & - & - & - & - \\
\hline Uncalcified fibrocartilage & $0.73 \pm 0.11$ & $0.76 \pm 0.11$ & $0.78 \pm 0.11$ & $0.76 \pm 0.11$ & $0.61 \pm 0.05$ & $0.63 \pm 0.05$ & $0.64 \pm 0.05^{\star \star}$ & $0.63 \pm 0.05^{*}$ \\
\hline Calcified fibrocartilage & $0.66 \pm 0.07$ & $0.68 \pm 0.07$ & $0.70 \pm 0.08$ & $0.68 \pm 0.07$ & $0.58 \pm 0.05$ & $0.60 \pm 0.05$ & $0.61 \pm 0.04^{\star \star}$ & $0.60 \pm 0.05^{*}$ \\
\hline Subchondral bone & $0.58 \pm 0.03$ & $0.56 \pm 0.02$ & $0.56 \pm 0.02$ & $0.57 \pm 0.03$ & $0.54 \pm 0.05$ & $0.54 \pm 0.04$ & $0.54 \pm 0.04$ & $0.54 \pm 0.06^{*}$ \\
\hline
\end{tabular}

Values are represented as mean \pm SD.

* Significantly different than the anterior attachment $(p<0.05)$.

** Significantly different than proximal region $(p<0.05)$. 
fibrocartilage interdigitates deeply within the subchondral bone at the cement line in meniscal attachment insertions. The interdigitations are calcified fibrocartilage like branch forms that go into the subchondral bone with the purpose to anchor the attachment into the bone. The interdigitations increase the surface area between the zones. It is believed that the number of interdigitations provide strength of the attachment. The posterior attachment was found to have a greater frequency and thickness of interdigitations compared to the anterior attachment. This correlated well with the fact that the posterior attachment had a greater ultimate strain compared to the anterior attachments. ${ }^{46}$ Not only may the interdigitations be responsible for the mechanical behavior, but the increased thicknesses of the zones in the posterior attachment may contribute to the increased ultimate strain seen in the posterior attachment compared to the anterior attachment. Both the frequency and thickness of the interdigitations was shown to increase from the proximal to distal regions of the medial meniscal attachment. This relates back to the fact that the proximal side of the external ligament attachment is longer than the distal side and may be related to the stress and strain levels seen in vivo.

Interestingly, for the anterior attachment it was shown that the proximal region (on average 20 crimps/ $\mathrm{mm}$ ) had a lower collagen crimp frequency than the distal region (on average 33 crimps $/ \mathrm{mm}$ ). Multiplying these numbers by the length of the external ligamentous portion of the attachment, which is different for the proximal and distal regions, results in 514 crimps in the proximal region and only 438 crimps in the distal region. Furthermore, for the posterior attachment, while differences were seen in the external ligamentous lengths for the proximal versus distal surface, no differences were seen in the average collagen crimp frequency between the proximal and distal regions, both were $27 \mathrm{crimps} / \mathrm{mm}$. This suggests that if the attachments are loaded uniformly in vivo, based on external lengths, more strain would be present on the distal surface. It does not appear that the distal surface has more crimp frequency to accommodate this. Meniscal attachments have a typical ligamentous structure, ${ }^{33}$ since they are mainly composed of Type I collagen. The tensile mechanical properties of soft connective tissues have been correlated with collagen crimping. ${ }^{36}$ The crimp pattern of collagen has been extensively studied, and some studies have shown that collagen fibrils start uncrimping at very low tensile loads ${ }^{10}$ and the toe region in the tensile stress-strain curve of ligaments and tendons is due to the straightening of crimped collagen fibrils. ${ }^{23}$ In a typical stress-strain curve for ligaments and tendons we can see three regions, these are: toe, heel, and linear. In the toe region, the strain increases with low stress, and the crimps straightened. ${ }^{13}$ At larger strains, passing through the heel and linear region, the collagen fibrils become straightened $^{23}$ and the stress has a significant increase compared with the first region, showing a relation between collagen crimping and bearing load of ligamentous structures. Care should be taken when interpreting data collected for strain measurements only on the proximal surface.

Surprisingly, GAG fraction in the meniscal attachments' insertion was significantly greater in the anterior attachment, while proteoglycans like aggrecan are usually associated with compressive strength. ${ }^{35}$ Since it was previously suggested that only the posterior attachment is exposed to compressive loads, we would have expected more GAGs in the posterior attachment. ${ }^{18}$ However, Type II collagen has been shown to be present in both medial meniscal attachments in the uncalcified fibrocartilage, calcified fibrocartilage, and the ligamentous zone $\mathrm{e}^{16}$; as well as aggrecan has been found in insertion sites of ligaments. ${ }^{34}$ Inasmuch as this type of collagen, usually found where aggrecan is present, ${ }^{27}$ is normally related with compression and shear forces, ${ }^{29,32}$ we might speculate that both insertion sites in meniscal attachments are subjected to tensile and compressive load in the loaded knee joint. It should be noted that our GAG fraction analysis is only semi-quantitative, comparing only relative amounts of GAG between regions and zones based on staining intensity. Additionally, the orientation of collagen bundles and surrounding GAG can influence the degree of GAG staining. ${ }^{45}$ Determination of GAG fraction was only semi-quantitative as it is not clear how to separate the zones to use methods other than staining to study GAG fraction. Care was taken to ensure that slices were of uniform thickness as this may affect staining intensity. Even though the same samples were used to determine both, the collagen crimp frequency was measured on the external visible ligamentous portion, and the GAG fraction was measured in the insertion zones. Therefore, it is likely that collagen crimp frequency did not affect GAG fraction on the insertion sites. The combined presence of GAGs found in this study and the Type II collagen previously found, does suggest that the attachments are subjected to compressive loads and warrants further investigation into the type of proteoglycans that are present in the attachments. Material subjected to compressive loads usually have high fraction of GAGs compared to materials primarily in tension (i.e., articular cartilage compared to a ligament). ${ }^{35}$ The mechanism of load transfer from the main body of the meniscus to the external ligamentous attachments into the bony insertion zones is unknown. 
Previous studies of meniscal attachments that have grouped all the attachments together to compare to the meniscus main body, indicate that the most abundant glycosaminoglycan in both meniscal attachments and the main body is chondroitin sulfate. ${ }^{1-3,37}$ The inner zone of the main body of the meniscus is thought to experience more compression and be composed of mostly chondrocytic-type cells whereas the outer zone is thought to be exposed to more tension and contain more fibroblastic type cells. ${ }^{30,44}$ This corresponds well with data showing that aggrecan was more concentrated in the inner versus the outer zone of the meniscus. ${ }^{45}$ We did not study the variation in GAG fraction from the attachment as it connects from the inner part of the meniscus to the outer part of the meniscus. Others have hypothesized that the proteoglycan content is related to the primary type of loading with more dermatan sulfate being present in regions of tension and chondroitin sulfate being found in larger quantities in regions of compression. ${ }^{1,21}$ It has been previously shown that the distribution and characteristics of the small proteoglycans in knee meniscus reflect regional adaptations to functional demands. ${ }^{38}$ Current research is underway to document the type of proteoglycans present in the various zones of the meniscal attachments.

While the current study is based on bovine attachments instead of human, this allows for comparison to mechanical property testing previously performed on bovine meniscal attachment tissue. ${ }^{31}$ Mechanically, both bovine tendon and bovine meniscal properties have been shown to correlate well to human properties. ${ }^{14,28,43}$ Thus, we might expect the biochemistry to be similar between bovine and human as well. However, a limitation of the bovine model, as well as the rabbit model, is that the posterior attachment of the lateral meniscus inserts into the femur, while in the human knee joint, this attachment inserts into the tibia.

Since meniscal attachments are important for meniscal function ${ }^{5,12,24}$ the description of meniscal attachment microstructure and composition could prove beneficial in the development of tissue-engineered menisci. Not only is the external ligamentous portion and bony insertions important, but future studies should consider the transition from a triangular fibrocartilage in the main body of the meniscus, to the mostly rectangular external ligamentous portion of the attachment.

\section{ACKNOWLEDGMENTS}

This study was supported in part by the Michigan Space Grant Consortium, the MTU SURF program, and the National Institutes of Health (AR051906-01).

\section{REFERENCES}

${ }^{1}$ Adams, M.E., and Y.A. Ho. Localization of glycosaminoglycans in human and canine menisci and their attachments. Connect Tissue Res. 16(3):269-279, 1987.

${ }^{2}$ Adams, M.E., C.A. McDevitt, A. Ho, and H. Muir. Isolation and characterization of high-buoyant-density proteoglycans from semilunar menisci. J. Bone Joint Surg. Am. 68(1):55-64, 1986.

${ }^{3}$ Adams, M.E., and H. Muir. The glycosaminoglycans of canine menisci. Biochem. J. 197(2):385-389, 1981.

${ }^{4}$ Ahmed, A.M., and D.L. Burke. In-vitro measurement of static pressure distribution in synovial joints-part i: tibial surface of the knee. J. Biomech. Eng. 105(3):216-225, 1983.

${ }^{5}$ Alhalki, M.M., S.M. Howell, and M.L. Hull. How three methods for fixing a medial meniscal autograft affect tibial contact mechanics. Am. J. Sports Med. 27(3):320-328, 1999.

${ }^{6}$ Ali, A.F., M.M. Taha, G.M. Thornton, N.G. Shrive, and C.B. Frank. Biomechanical study using fuzzy systems to quantify collagen fiber recruitment and predict creep of the rabbit medial collateral ligament. J. Biomech. Eng. 127(3):484-493, 2005.

${ }^{7}$ Atkinson, T., and R. C. Haut. The influence of collagen fiber orientation on tendon creep and relaxation, Tran. ORS, 46th annual meeting, p. 779, 2000.

${ }^{8}$ Benjamin, M., E.J. Evans, R.D. Rao, J.A. Findlay, and D.J. Pemberton. Quantitative differences in the histology of the attachment zones of the meniscal horns in the knee joint of man. J. Anat. 177:127-134, 1991.

${ }^{9}$ Benjamin, M., and J.R. Ralphs. Fibrocartilage in tendons and ligaments - an adaptation to compressive load. J. Anat. 193(Pt 4):481-494, 1998.

${ }^{10}$ Boorman, R.S., T. Norman, F.A. Matsen, 3rd, and J.M. Clark. Using a freeze substitution fixation technique and histological crimp analysis for characterizing regions of strain in ligaments loaded in situ. J. Orthop. Res. 24(4):793799, 2006.

${ }^{11}$ Burks, R.T., M.H. Metcalf, and R.W. Metcalf. Fifteenyear follow-up of arthroscopic partial meniscectomy. Arthroscopy 13(6):673-679, 1997.

${ }^{12}$ Chen, M.I., T.P. Branch, and W.C. Hutton. Is it important to secure the horns during lateral meniscal transplantation? A cadaveric study Arthroscopy 12(2):174-181, 1996.

${ }^{13}$ Diamant, J. Collagen; Ultrastructure and its Relation to Mechanical Properties as a Function of Ageing. Proc. $R$. Soc. Lond. Ser-B 180(60):293-315, 1972.

${ }^{14}$ Donahue, T.L., C. Gregersen, M.L. Hull, and S.M. Howell. Comparison of viscoelastic, structural, and material properties of double-looped anterior cruciate ligament grafts made from bovine digital extensor and human hamstring tendons. J. Biomech. Eng. 123(2):162-169, 2001.

${ }^{15}$ Englund, M., E.M. Roos, H.P. Roos, and L.S. Lohmander. Patient-relevant outcomes fourteen years after meniscectomy: Influence of type of meniscal tear and size of resection. Rheumatology (Oxford) 40(6):631-639, 2001.

${ }^{16} \mathrm{Gao}, \mathrm{J}$. Immunolocalization of types i, ii, and $\mathrm{x}$ collagen in the tibial insertion sites of the medial meniscus. Knee Surg. Sport Traumatol. Arthrosc. 8(1):61-65, 2000.

${ }^{17} \mathrm{Gao}, \mathrm{J}$. , and K. Messner. Quantitative comparison of soft tissue-bone interface at chondral ligament insertions in the rabbit knee joint. J. Anat. 188(Pt 2):367-373, 1996.

${ }^{18}$ Gao, J., G. Oqvist, and K. Messner. The attachments of the rabbit medial meniscus. A morphological investigation 
using image analysis and immunohistochemistry. J. Anat. 185(Pt 3):663-667, 1994.

${ }^{19}$ Gao, J., T. Rasanen, J. Persliden, and K. Messner. The morphology of ligament insertions after failure at low strain velocity: An evaluation of ligament entheses in the rabbit knee. J. Anat. 189 (Pt 1):127-133, 1996.

${ }^{20}$ Gao, J., X. Wei, and K. Messner. Healing of the anterior attachment of the rabbit meniscus to bone. Clin. Orthop. (348):246-58, 1998.

${ }^{21}$ Gillard, G.C., H.C. Reilly, P.G. Bell-Booth, and M.H. Flint. The influence of mechanical forces on the glycosaminoglycan content of the rabbit flexor digitorum profundus tendon. Connect Tissue Res. 7(1):37-46, 1979.

${ }^{22}$ Goertzen, D., J. Gillquist, and K. Messner. Tensile strength of the tibial meniscal attachments in the rabbit. J. Biomed. Mater. Res. 30(1):125-128, 1996.

${ }^{23}$ Hansen, K.A., J.A. Weiss, and J.K. Barton. Recruitment of tendon crimp with applied tensile strain. J. Biomech. Eng. 124(1):72-77, 2002.

${ }^{24}$ Haut Donahue, T.L., M.L. Hull, M.M. Rashid, and C.R. Jacobs. How the stiffness of meniscal attachments and meniscal material properties affect tibio-femoral contact pressure computed using a validated finite element model of the human knee joint. J. Biomech. 36(1):19-34, 2003.

${ }^{25}$ Heinegård, D., and A. Oldberg. Glycosylated matrix proteins. In: Connective Tissue and its Heritable Disorders, edited by P.M. Royce and B. Steinmann. New York: Wiley Liss, 1993, pp. 189-209.

${ }^{26}$ Huang, Q., D. Opstelten, N. Samman, and H. Tideman. Experimentally induced unilateral tooth loss: Histochemical studies of the temporomandibular joint. J. Dent. Res. 81(3):209-213, 2002.

${ }^{27}$ Ilic, M.Z., P. Carter, A. Tyndall, J. Dudhia, and C.J. Handley. Proteoglycans and catabolic products of proteoglycans present in ligament. Biochem. J. 385(Pt 2):381-388, 2005.

${ }^{28}$ Joshi, M.D., J.K. Suh, T. Marui, and S.L. Woo. Interspecies variation of compressive biomechanical properties of the meniscus. J. Biomed. Mater. Res. 29(7):823-828, 1995

${ }^{29}$ Kadler, K.E., D.F. Holmes, J.A. Trotter, and J.A. Chapman. Collagen fibril formation. Biochem. J. 316(Pt 1):1-11, 1996.

${ }^{30}$ LeRoux, M.A., and L.A. Setton. Experimental and biphasic fem determinations of the material properties and hydraulic permeability of the meniscus in tension. J. Biomech. Eng. 124(3):315-321, 2002.

${ }^{31}$ Maes, J.A., and T.L. Haut Donahue. Time dependent properties of bovine meniscal attachments: Stress relaxation and creep. J. Biomech. 39(16):3055-3061, 2006.

${ }^{32}$ Merrilees, M.J., and M.H. Flint. Ultrastructural study of tension and pressure zones in a rabbit flexor tendon. Am. J. Anat. 157(1):87-106, 1980.

${ }^{33}$ Messner, K., and J. Gao. The menisci of the knee joint. Anatomical and functional characteristics, and a rationale for clinical treatment. J. Anat. 193(Pt 2):161-7178, 1998.
${ }^{34}$ Moriggl, B., P. Jax, S. Milz, A. Buttner, and M. Benjamin. Fibrocartilage at the entheses of the suprascapular (superior transverse scapular) ligament of man-a ligament spanning two regions of a single bone. J. Anat. 199(Pt 5):539-545, 2001.

${ }^{35}$ Mow, V.C., W. Zhu, and A. Ratcliffe. Structure and function of articular cartilage and meniscus. In: Basic Orthopaedic Biomechanics, edited by V.C. Mow and W.C. Hayes. New York: Raven Press, 1991.

${ }^{36}$ Nordin, M., and V.H. Frankel. Basic Biomechanics of the Musculoskeletal System. Lea \& Fibiger: Philadelphia, 1980.

${ }^{37}$ Roughley, P.J., and R.J. White. The dermatan sulfate proteoglycans of the adult human meniscus. J. Orthop. Res. 10(5):631-637, 1992.

${ }^{38}$ Scott, P.G., T. Nakano, and C.M. Dodd. Isolation and characterization of small proteoglycans from different zones of the porcine knee meniscus. Biochim. Biophys. Acta 1336(2):254-262, 1997.

${ }^{39}$ Setton, L.A., F. Guilak, E.W. Hsu, and T.P. Vail. Biomechanical factors in tissue engineered meniscal repair. Clin. Orthop. (367 Suppl):S254-S272, 1999.

${ }^{40}$ Shoemaker, S.C., and K.L. Markolf. The role of the meniscus in the anterior-posterior stability of the loaded anterior cruciate-deficient knee. Effects of partial versus total excision. J. Bone Joint Surg. Am. 68(1):71-79, 1986.

${ }^{41}$ Smith, G.N., E.A. Mickler, M.E. Albrecht, S.L. Myers, and K.D. Brandt. Severity of medial meniscus damage in the canine knee after anterior cruciate ligament transection. Osteoarthr. Cartilage 10(4):321-326, 2002.

${ }^{42}$ Sweigart, M.A., and K.A. Athanasiou. Toward tissue engineering of the knee meniscus. Tissue Eng. 7(2):111-129, 2001.

${ }^{43}$ Sweigart, M.A., C.F. Zhu, D.M. Burt, P.D. DeHoll, C.M. Agrawal, T.O. Clanton, and K.A. Athanasiou. Intraspecies and interspecies comparison of the compressive properties of the medial meniscus. Ann. Biomed. Eng. 32(11):15691579, 2004.

${ }^{44}$ Upton, M.L., A. Hennerbichler, B. Fermor, F. Guilak, J.B. Weinberg, and L.A. Setton. Biaxial strain effects on cells from the inner and outer regions of the meniscus. Connect Tissue Res. 47(4):207-214, 2006.

${ }^{45}$ Valiyaveettil, M., J.S. Mort, and C.A. McDevitt. The concentration, gene expression, and spatial distribution of aggrecan in canine articular cartilage, meniscus, and anterior and posterior cruciate ligaments: a new molecular distinction between hyaline cartilage and fibrocartilage in the knee joint. Connect Tissue Res. 46(2):83-91, 2005.

${ }^{46}$ Villegas, D.F., J.A. Maes, S.D. Magee, and T.L. Haut Donahue. Failure properties and strain distribution analysis of meniscal attachments. J. Biomech. 40(12):26552662, 2007

${ }^{47}$ Woo, S.L., G.A. Livesay, T.J. Runco, and E.P. Young. Structure and function of tendons and ligaments. In: Basic Orthopaedic Biomechanics, edited by V.C. Mow and W.C. Hayes. Philadelphia: Lippincott-Raven, 1997, pp. 209-252. 